reducing the concern over a hypothetical future event. Both groups have non-scientific motives in their baggage. At least in Europe, the first group dominates, and it may indeed be very helpful to read what the other faction of scientists have to say.

The book's last section deals with the social process of science, how certain views, such as the probability that global warming will have disastrous effects, become dominating paradigms and how the organization of science may influence this process. These are relevant questions, and research agencies would do well to provide more funding for interdisciplinary research into such matters, particularly if they feed directly into policymaking.

The book is well written and easy to read. And the authors should be applauded for their bold predictions for the future, which will be proven right or wrong by the course of history: the Kyoto Protocol will have no effect; carbon dioxide emissions will continue to increase; by 2050, the Earth's average surface temperature will have increased by $0.65-$ $0.75^{\circ} \mathrm{C}$ in the winter half-year and by between 0.60 and $0.65{ }^{\circ} \mathrm{C}$ in summer; by 2050, crop yields will have risen sufficiently for the (carbon dioxide-related) rise alone to feed one-quarter of today's population; temperature-related mortality will decline.

Others make different forecasts. Hans von Storch is at the Institute of Hydrophysics, GKSS Research Centre, Max-Planck-Strasse 1, PO Box, 21502 Geestacht, Germany.

\section{To the core of consciousness}

\section{A Universe of Consciousness: How Matter Becomes Imagination/ Consciousness: How Matter Becomes Imagination \\ by Gerald Edelman \& Giulio Tononi Basic Books/Allen Lane: 2000. 288 pp. $\$ 27.50 / £ 20$}

\section{Raymond J. Dolan}

A Universe of Consciousness is the latest in a series of books in which the principal author, Gerald Edelman, develops a neurobiological theory of consciousness. It is no exaggeration to say that his previous books have aroused conflicting reactions. A frequent criticism has been that he couches fairly simple ideas in an idiosyncratic jargon that renders the end product impenetrable. Such criticism will be hard to level against the current volume, coauthored by Guilio Tononi, which represents the best and most accessible introduction to Edelman's ideas on consciousness.

A substantial portion of the book goes over familiar ground. In brief, Edelman has championed a view that brain function and organization are determined by Darwinian evolutionary principles that occur within the lifetime of the organism. Consequently, major sections are taken up with accounts of selectionism, the concept of value and the

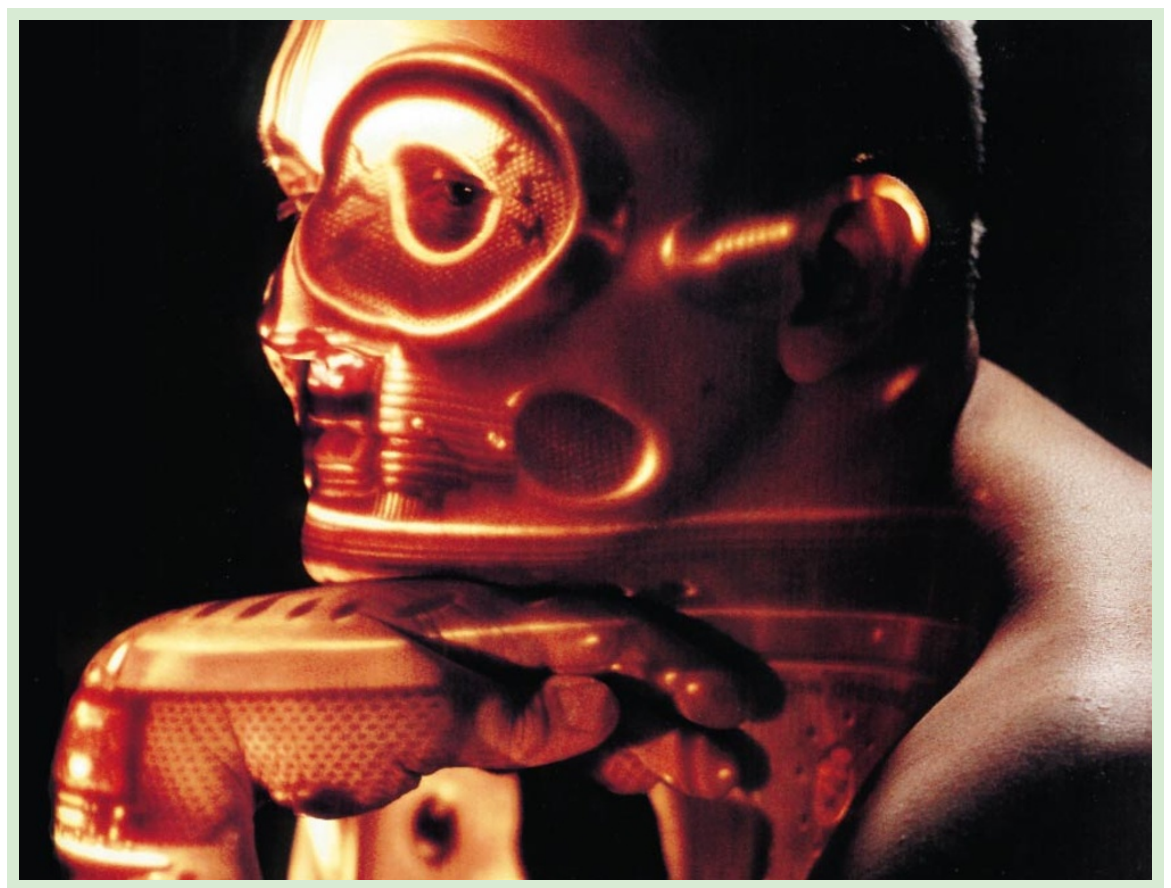

\title{
Man made machine?
}

Is it possible that we will eventually become robots ourselves, uploading our consciousness into computers? Robo Sapiens: Evolution of a
New Species by Peter Menzel and Faith D’Aluisio (MIT Press, \$29.95, £19.95) shows how far we have come along that path. critical role of neuronal interactions mediated by a process he has labelled re-entry. Reentry is a much misunderstood idea, often confused with feedback. It refers to the dynamic interchange between reciprocally connected brain regions that is fundamental to synchronization and the coordination of their mutual functions.

All of these ideas are treated clearly and concisely. There is also wide acknowledgement of competing ideas on the structure of consciousness and, although it is clear that the main issue is higher-order consciousness, there is an acceptance that a complete account will include what has been termed primary consciousness.

A Universe of Consciousness still provides its moments of frustration. A prime example is the treatment of memory, in which the authors propose the controversial idea that all forms of memory are non-representational - here the authors provide the useful geological metaphor that memory is more like the melting and freezing of a glacier (non-representational) than an inscription on a rock (representational). In their account, memory results from selective matching between distributed neural activity and signals from the world, the body and the brain itself. But this potentially radical proposal is not developed sufficiently.

The book's novel contribution is the development of what is termed the "dynamic core hypothesis". Put simply, a dynamic core is a subset of brain regions identified by the pattern of neuronal interactions within the core and its associated regions. As an analogy, the core can be considered akin to a shoal of fish. The envelope, or boundary, is clearly discernible but continuously changes as the fish (neuronal activity) swim to different locations (different neuroanatomical domains). The fish swim in concert, but each has its own unique trajectory through the water (that is, neuronal activity is coherent but not identical).

Critically, the core is characterized by being functionally disconnected from the rest of the brain. This disconnection is represented by relatively independent neuronal dynamics inside and outside the core. Thus, the authors liken the dynamic core to a functional cluster. The second key idea is that patterns of activity within the core exhibit a high degree of complexity. The complexity of the core is important because it reflects a high degree of differentiation, implicit in observing a particular brain state, given the diversity of patterns that could be expressed. The boundaries that define the core are predicated on constantly changing patterns of activity and are themselves dynamic, reflecting the functional state of the brain.

The notion of a dynamic core is both conceptually appealing and usefully grounded in information theory. There is, however, a problem with it. This arises from the fact that 
it blends the theory of dynamical systems with information theory relating to stationary stochastic systems. The problem can be seen by considering a dynamic core over a short period of time. The complexity within this time frame is represented by the shape of the probability distribution of the activities of the elements comprising the core. The question is: what does this probability distribution refer to? Does it refer to realizations of brain activity patterns over time or to different realizations at the particular time that that core exists?

The authors have assumed the former when measuring complexity using neuroimaging time series. However, it cannot be applied to the dynamic core hypothesis because any particular core is defined only over a short period of time. If the core differentiates between a vast repertoire of potential states by expressing just one state during its transient existence, then it cannot be complex, because the repertoire of states within its lifetime must, by definition, be severely restricted. If it were not restricted, no differentiation would occur and there would be no information about which brain state had been selected.

The second possibility, that complexity refers to realizations at the same point in time, is more tenable. And it could be linked to work on dynamic correlations that employs multiple trials to look at the correlations among neurons as a function of peristimulus time. However, this formulation precludes the existence of a unique dynamic core that exists only once in the lifetime of the organism (that is, conscious states must be reiterated). In short, by basing the definition of a dynamic core on the probability distribution of its underlying activity the authors imply that there is a repertoire of potential realizations that engender this probability distribution. Because the dynamic core is transient, with continuously changing boundaries, these realizations cannot be over time. Therefore, unless the same dynamic core is recapitulated exactly, on many different occasions, the probability distribution, on which its definition rests, does not exist.

Despite this criticism, the dynamic core hypothesis is a highly compelling idea. One can easily see how such ideas would flourish in the context of dynamical systems theory, where the creation of information and measurement of entropy in nonlinear dynamical systems are natural consequences of sensitivity to initial conditions.

The authors' proposal that consciousness is based on distributed neural activity necessarily rules out any attempt to assign a different neural group to every phenomenological state. When translated into neuronal terms, this enterprise rapidly runs up against a problem of scale, given that the approach requires that every property is represented by a distinct neural group. The question posed by the authors is: why should we suppose that firing in any particular group of neurons is associated with a specific subjective quality? In their own words, "the enormous petitio principiiso unveiled ... would trigger a sudden fit of inextinguishable laughter in the Olympians".

Explaining consciousness has become the Holy Grail of modern neuroscience. Any reckoning on who has found the true path is surely premature. Nevertheless, the account of consciousness provided by Edelman and Tononi is certainly highly plausible and can be recommended as one of the most ambitious accounts around.

Raymond J. Dolan is in the Wellcome Department of Cognitive Neurology, Institute of Neurology, London WC1N 3BG, UK.

\section{$\bullet \bullet \bullet \bullet \bullet \bullet \bullet \bullet \bullet \bullet \bullet \bullet \bullet \bullet \bullet \bullet \bullet \bullet \bullet \bullet \bullet \bullet$ A dip in the soup, with a pinch of salt}

The Spark of Life: Darwin and the Primeval Soup

by Christopher Wills \& Jeffrey Bada

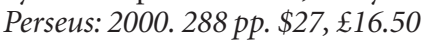

\section{William J. Hagan}

Conventional images of the early Earth portray a hostile planet with violent displays of lightning and volcanic eruptions. To these pyrotechnics, one might add the occasional asteroid impact and the smoky plumes of hydrothermal vents. Beyond providing melodrama for artistic depictions (including those reproduced in this book by Bill Hartmann of the University of Arizona), such phenomena suggest possible energy sources that may have played a role in the origin of life. In The Spark of Life Christopher Wills and Jeffrey Bada review our understanding of the origins of life for the informed general reader.

There has been a lot of interest in evaluating the relative importance of different routes towards the organic precursors of the first life. One strategy is to assess the fluxes of energy on the modern Earth, then to estimate the evolution of these energy sources through time, and finally, to determine the relative rates of formation and decomposition of potentially prebiotic compounds. Although the first task should be the most straightforward, the compilation of energy inputs for the modern Earth has been complicated by shortage of data and by propagation of values from the older literature. Chris Chyba and Carl Sagan pointed out in 1991, for example, that the rate of terrestrial lightning dissipation is about 20 -fold lower than previous estimates.

Controversy (often acrimonious) has also centred on the role of hydrothermal vents in prebiotic synthesis, since their discovery two decades ago. Although the large temperature gradients and hydrogen-rich conditions make them attractive to those (called "ven-

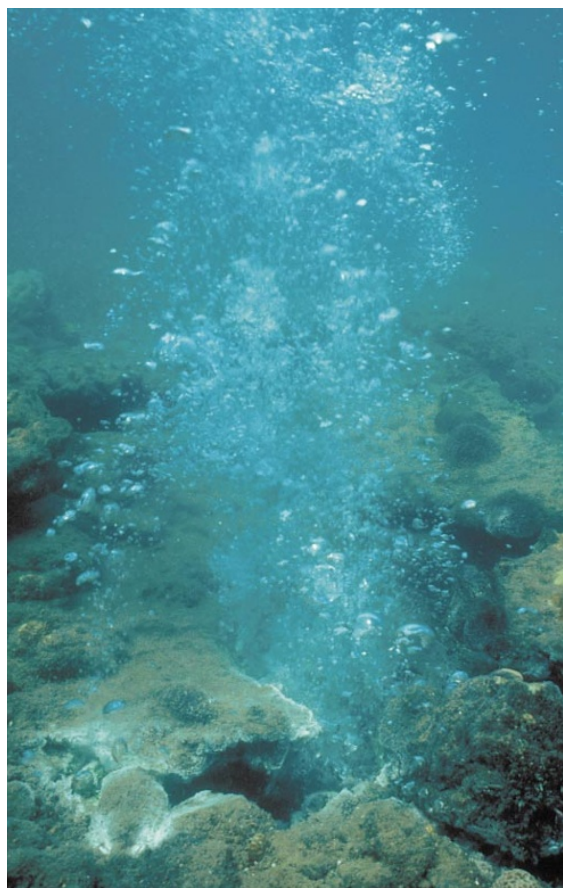

Murky birth? 'Ventists' believe that the precursors to life may have originated in hydrothermal vents.

tists" in this book) who believe that vents played a major role, critics, including one of the authors, have stressed the sterilizing effect of marine cycling through these hot regions. Agreement has been hampered both by the inaccessibility of these deep environments, and by the difficulties of laboratory simulation. The latter challenge is being addressed by several research groups, whose data it is hoped will confirm the results of thermodynamic calculations. Even if vents did facilitate the formation of small biomolecules, the transient lifetime of particular smokers makes it unlikely that a sustained sequence of reactions leading to protobionts could occur there; these organic sources may have simply augmented the overall concentration of the primordial soup.

The role of sunlight in prebiotic synthesis has become less fashionable, but any ranking of energy sources always puts ultraviolet and visible radiation at the top. Calculating the distribution of sunlight on the early Earth is complicated by the fact that, during the Sun's T-Tauri stage 4.6 billion years ago, its output was about 30\% dimmer and ultraviolet light was more intense. Nevertheless, the total flux at wavelengths less than 230 nanometres 4.2 billion years ago is comparable to that reaching the edge of the Earth's atmosphere today, and this energy could have played a role in the formation of small precursors such as formaldehyde. Although longer wavelengths would probably have penetrated to the surface (especially in the absence of an ozone shield), solar-driven processes in this spectral regime have been less extensively investigated in the context of the origins of life.

The Spark of Life takes a sceptical view of 